liche" Linke. Der „Lange Marsch" wird dort auch als erfolgreicher "strategischer Rückzug" bezeichnet ${ }^{2}$.

Offensichtlich zielt Braun in seinen Memoiren darauf ab, die Geschichte der KP Chinas den neuen politischen Richtlinien in der DDR und der Sowjetunion anzupassen. Wir wissen, daß sich Peking und Moskau in ihren ideologischen Auseinandersetzungen ständig darum bemühen, einander des Großmachtchauvinismus $\mathrm{zu}$ bezichtigen. Es ist auch nicht unbekannt, daß eine Kluft zwischen der Komintern und der KP Chinas unter Mao Tse-tung bestand. Dies ist nicht weiter verwunderlich; denn Stalin hatte die Komintern des öfteren als Instrument der sow jetischen Außenpolitik eingesetzt. Ausschließlich im sowjetischen Interesse, um nämlich gegenüber den Westmächten die Macht der Sowjetunion harmloser erscheinen $\mathrm{zu}$ lassen und dadurch nationale Nachkriegspläne realisierbar zu machen, wurde diese Internationale im Mai 1943 aufgelöst. Deshalb scheint Otto Brauns Kritik an Maos Beziehungen zu Moskau und der Komintern auch zu einseitig.

Ein anderer Mangel an Brauns Memoiren liegt darin, daß der Autor eine lange Geschichte in kurze Form gefaßt hat. Notwendigerweise sind seine Schilderungen deshalb ziemlich grob. Auch seine eigene Verbindungsfunktion $z$ wischen der Komintern und der KP Chinas sowie sein Verkehr mit den chinesischen kommunistischen Führern ist leider zu kurz gekommen. Wie er selbst zugibt, kennt er die chinesische Sprache nicht. Es ist dem Leser an vielen Stellen unklar, wie Braun trotz seiner Sprachschwierigkeiten und trotz des Mißtrauens Mao Tse-tungs gegenüber der Komintern dessen wichtige Entscheidungen einschließlich der noch nicht bekanntgegebenen Pläne und Gedanken erlebt hat.

Um Brauns Memoiren zu vergleichen, seien dem Leser das Buch von Edgar Snow "Red Star over China" (London 1938) und Chang Kuo-t'aos Memoiren (ab März 1966 in einer chinesischen Zeitschrift "Ming $\mathrm{Pao}$ " in Hongkong fortsetzungsweise veröffentlicht) empfohlen. Snow besuchte 1936 das kommunistische Gebiet in Nordshensi und bringt in dem Buch seine Reise und Interviews bei zahlreichen chinesischen kommunistischen Führern vor. Chang war früher ein bedeutender kommunistischer Führer und hat sogar an der Gründung der KP Chinas teilgenommen. Er kehrte 1938 der Partei den Rücken, wohnte lange Zeit in Hongkong und lebt jetzt in Kanada.

Y. H. N i e h

\section{Chitoshi Yanaga}

Big Business in Japanese Politics

Yale University Press

New Haven and London 1968

IX +371 Seiten.

Wer sich gründlich und im einzelnen über Staats- und Verfassungswirklichkeit in ihrem entscheidenden Punkte, dem Verhältnis von Wirtschaft und Politik, im Hinblick auf Japan zu unterrichten wünscht, wird in diesem Buch eine vortreffliche Erfüllung solcher Wünsche finden. Grundlage sowohl dieses Verhältnisses wie für dessen Verständnis sind die zwischenmenschlichen Beziehungen und die spezifisch gesellschaftlichen Gruppen (12). Hierher gehört als erstes die Familie, die ist "still the touchstone of social success ... More than ever, family status (iyegara) and pedigree (kenami) are necessary qualifications for membership in high society and for achieving a position of prestige and influence in business and politics, even in academic life" (15). Es nimmt nicht mehr wunder, daß auch die Heirat von solchen Gesichtspunkten beherrscht wird: S. 327 gibt der Verfasser eine Liste unter der bezeichnenden Uberschrift „Marital Network Linking Business, Political and Academic Elites with the Imperial Family and the Former Aristocracy". Außer 
der Familie gibt es alle möglichen Cliquen, die auf Gemeinsamkeit von Schuloder Universitätsbesuch, landschaftlichen und anderen Grundlagen beruhen. Insbesondere ist in diesem Zusammenhang auch die Bürokratie zu nennen. Dies alles bedeutet: „The Interpersonal Relationship is the Matrix of Business and Politics" (12).

Auf solcher gesellschaftlich-personaler Grundlage existiert "the governing process esentially" als "the joint effort of big business, party \& government, and the bureaucratic leadership" (VIII; 27). Wer in diesem Prozeß die Vorherrschaft innehat, ist nicht ohne weiteres klar: "It is impossible to determine exactly, how and by whom the country is governed" $(26 ; 34)$. Gewiß kommt ein solcher Vorrang nicht der Legislative $\mathrm{zu}$ (über den Einfluß von big business auf sie vgl. S. 307); denn wie herkömmlich wird der Exekutive der Vorrang vor der Legislative gegeben (103). Die Exekutive jedoch steht erst recht unter dem Einfluß von big business: So bestimmt "Zaikai" (= big business power group) über Leben und Tod der Regierung $(7 ; 32 / 33 ; 141 ; 148)$; es ist in derselben durch mehrere Mitglieder maßgebend vertreten (70; 72 f.); mittelbar wird diese Herrschaft gestützt durch die Abhängigkeit der politischen Parteien (Überblick über die Spenden S. 80) und der Wirtschaftsverbände von big business (55). Insgesamt ergibt sich: Von den drei Kräften, die Japan regieren, ist "the role of big business in this triumvirate unquestionably far more important today than before the war" (34).

Diese Vorherrschaft der Wirtschaft in der staatlichen Dreiheit bedeutet aber nicht Ókonomisierung der Politik. Die letzten Ziele auch von big business nämlich sind nicht ökonomische, sondern politische, angefangen von der Lebensfähigkeit der Nation, die angesichts der Kargheit der natürlichen Ausstattung "must trade or perish". Bezeichnend für eine solche nationale Orientierung ist es, daß Zaikai wünscht ein "strong and effective government" (145) und Planung in einer solchen Situation für unerläßlich hält (310 f.). Wirtschaft und Staat ziehen somit am gleichen Strang, und hierin liegen sowohl Voraussetzung wie Notwendigkeit der vom Verfasser immer beobachteten Zusammenarbeit zwischen beiden.

Der hohe Wert des Werkes wird gesteigert durch Exkurse, die Inzidentthemen gründlich und ergiebig behandeln. Genannt seien als solche Exkurse die $\mathrm{Ka}$ pitel 4 "The Role of Bureaucracy “ und 8 "Southeast Asia Reparations Settlement". Das letzte zeigt, daß der Sinn der Reparation hier nicht Strafe oder Schadensersatz, sondern Einleitung von internationaler wirtschaftlicher Zusammenarbeit zwischen Besiegten und Siegern ist. Es ist unter solchen Vorzeichen nicht paradox, wenn die japanische Wirtschaft zum Abschluß entsprechender Abkommen drängt, bis zu dem extremen Ergebnis (227), daß sogar dort Reparationen geleistet werden, wo gar kein Schaden entstanden ist, Reparation also in Entwicklungshilfe übergeht.

Herbert Krüger

\section{Hans Wilhelm Vahlefeld 100 Millionen Außenseiter \\ (Die neue Weltmacht Japan) \\ Econ Verlag Düsseldorf/Wien 1969}

Das anzuzeigende Werk gehört zu jener Art von Sachbüchern, welche nicht nur ihrem Gegenstand gerecht werden, sondern auch ihrem Leser entgegenkommen wollen. Als es geschrieben wurde, hatte der unvergleichliche Aufstieg Japans in den internationalen Produktionsstatistiken der politisch interessierten Offentlichkeit in der westlichen Welt zum Bewußtsein gebracht, daß sie ihre Aufmerksamkeit für die Entwicklung im fernen Osten viel zu sehr auf Indien und Rotchina konzentriert habe. Gewohnt, die Probleme der Dritten Welt als Entwicklungsprobleme zu sehen, die zwar manchmal das Gewissen, doch nicht das Selbstbewußtsein der westlichen Industriegesellschaft anrührten, brachten diese dem Unverhofften eine sichtlich von Besorgnis gespeiste Neugier entgegen. Diese Neugier will der Autor mit seinem Buch befriedigen. 